\title{
Tangence
}

\section{Le lecteur modèle, instance de réalisation du lecteur empirique1}

\section{Noëlle Sorin}

Numéro 67, automne 2001

L'écriture pour la jeunesse : de la production à la réception

URI : https://id.erudit.org/iderudit/009617ar

DOI : https://doi.org/10.7202/009617ar

Aller au sommaire du numéro

\section{Éditeur(s)}

Presses de l'Université du Québec

ISSN

0226-9554 (imprimé)

1710-0305 (numérique)

Découvrir la revue

Citer cet article

Sorin, N. (2001). Le lecteur modèle, instance de réalisation du lecteur empirique1. Tangence, (67), 81-95. https://doi.org/10.7202/009617ar
Résumé de l'article

Résumé

L'esthétique de la littérature pour la jeunesse est devenue une esthétique de la communication par laquelle l'auteur est tenu d'ajuster son texte au lecteur, en se pliant ainsi aux exigences du marché. Mais malgré des efforts louables pour rendre la littérature accessible au plus grand nombre, auteurs et maisons d'édition, plutôt que d'amener les jeunes à la littérature, ont amené la littérature aux jeunes en opérant une sorte de nivellement par le bas du fait littéraire. À partir d'oeuvres de qualité, parfois même exi- geantes pour le jeune lecteur, une didactique de la littérature comprenant

l'enseignement/apprentissage à la fois de la lecture et de l'écriture littéraires serait une avenue prometteuse de déve- loppement de la compétence culturelle des élèves en réduisant l'écart entre le rôle du lecteur tel qu'institué par le texte et le lecteur empirique. 


\section{Le lecteur modèle, instance de réalisation du lecteur empirique ${ }^{1}$}

\section{Noëlle Sorin, Université du Québec à Trois-Rivières}

Depuis quelques décennies, la tendance est de contraindre la littérature pour la jeunesse à se plier aux besoins du lecteur empirique, c'est-à-dire du sujet concret de l'acte de lecture, le jeune lecteur en chair et en os, ce qui la confine à des productions dites de masse, souvent de moindre qualité. Or, le Programme de formation de l'école québécoise ${ }^{2}$, actuellement en implantation au primaire, réaffirme la dimension culturelle des visées éducatives. Celle-ci est notamment associée au développement d'une compétence culturelle qu'alimenterait particulièrement la fréquentation d'œuvres littéraires. Cette préoccupation nous invite à réfléchir sur la place et les fonctions de la littérature en didactique du français au primaire et à appréhender la construction du littéraire comme objet de connaissance. En l'état actuel des choses, nombre d'ceuvres de la littérature pour la jeunesse, à cause de cette propension qu'ont les auteurs et les maisons d'édition à vouloir les adapter au jeune public lecteur, s'éloignent de la littérarité et répondent mal aux besoins des enseignants qui désirent véritablement travailler sur des textes comportant une dimension littéraire en classe de français.

Toute activité interprétative est réglée par le texte, qui porte en quelque sorte en lui les prescriptions de sa propre lecture à travers cette stratégie textuelle que résume l'idée de "Lecteur modèle ", concept qui est emprunté ici à Umberto Eco ${ }^{3}$. Ces modalités textuelles prescrivent les réactions du lecteur empirique

1. Cet article est en partie inspiré de Noëlle Sorin, La lisibilité dans le roman pour enfants de 10-12 ans par une analyse sémiotique des textes, thèse de doctorat, 2 tomes, Université du Québec à Montréal, 1996, 596 f. Depuis 1999, notre thèse peut être consultée dans "Didactique et Acquisition du Français langue maternelle (DAF), base de données informatisée.

2. Ministère de l'Éducation du Québec (MEQ), Programme de formation de l'école québécoise, Québec, Gouvernement du Québec, 2001.

3. Umberto $\mathrm{Eco}$, Lector in fabula ou La coopération interprétative dans les textes narratifs [1979], traduction de l'italien par Myriem Bouzaher, Paris, Grasset et Fasquelle, 1985. 
et celui-ci, en tant que sujet concret de cet acte de coopération qu'est la lecture, doit se réaliser comme Lecteur modèle. Nous reviendrons plus avant sur ce concept, car c'est le rôle de l'enseignant de guider le lecteur empirique dans cette démarche grâce à l'enseignement/apprentissage de la littérature et la mise en jeu de ces pratiques que sont la lecture et l'écriture littéraires.

\section{Le contexte éducationnel}

L'enseignement de la littérature, dès le début de la scolarité (surtout aux deuxième et au troisième cycle du primaire), est désormais envisageable, d'autant plus qu'en ce tournant de siècle, la réforme de l'éducation entreprise à la suite des États généraux tenus en 1995-1996 a notamment mis l'accent sur l'importance de la lecture et de la connaissance des textes littéraires dans l'apprentissage du français, langue d'enseignement, et sur le développement d'une compétence culturelle qui doit mettre à portée $\mathrm{d}^{\prime}$ "apprécier des ceuvres littéraires ${ }^{4}$ ". Comme en font foi les nombreuses recherches et applications pratiques de ces dernières années, on ne confond plus l'enseignement du français et celui de la littérature, et on ne pose plus comme préalable à l'apprentissage de la littérature celui de la langue et de la grammaire. Par ailleurs, cette compétence en vertu de laquelle on se retrouve à même d'apprécier des ouvres littéraires est le lieu d'intégration par excellence des autres compétences langagières, puisque l'élève peut y réinvestir ses acquis en lecture, en écriture et en communication orale. De fait, "l'appréciation des cuvres littérai-

4. MEQ, Programme de formation de l'école québécoise, ouvr. cité. Voici l'explicitation ministérielle du sens de cette compétence (p. 84):

La fréquentation régulière d'ceuvres de qualité permet à lá fois de mieux se connaître et de nieux comprendre les autres et le monde environnant. Flle enrichit la langue et les connaissances générales cle l'élève en plus d'alimenter son imaginaire, de stimuler sa créativité et de développer son sens critique. La clécouverte progressive de ces ouvres amène chaque individu à identifier ses préférences et ses intérêts tout en lui offrant un lieu privilégié de réinvestissement de ses acquis en lecture, en écriture et en communication orale. Il se construit ainsi un réseau de repères culturels qu'il enrichit au cours d'expériences diversifiées et au contact d'autrui. Il apprend aussi à se doter de critères pour poser des jugements critiques et esthétiques sur les cuvres ainsi que pour justifier ses appréciations. En comparant ses perceptions et ses jugements avec ceux de ses pairs, il est parfois ámené à les enrichir, à les reconsidérer ou à les nuancer. 
res sollicite la majorité des compétences transversales et des domaines généraux de formation. Elle contribue de façon particulière à nourrir l'identité personnelle et culturelle de l'élève en suscitant l'exercice de sa pensée créatrice et de son jugement critique ${ }^{5}$.

Toutefois, la littérature entendue comme discipline scolaire avec ses objets d'apprentissage et ses méthodes d'enseignement est véritablement à la recherche d'une didactique au niveau primaire. Celle-ci s'articulerait autour de trois dimensions : la dimension épistémologique (la construction d'un objet de savoir), la dimension psychosociologique (l'appropriation de cet objet de savoir par les élèves) et la dimension praxéologique (les pratiques d'enseignement et d'apprentissage de cet objet de savoir). L'objet de savoir que constitue la littérature procéderait non pas de son histoire comme champ ou comme institution, mais plutôt des biens et des pratiques qui la composent ${ }^{6}$, notamment la lecture et l'écriture littéraires. La lecture littéraire ${ }^{7}$ est à la fois participative, car relevant de l'affect, et distanciée, c'est-à-dire commandant un travail cognitif sur le texte et son fonctionnement. L'écriture littéraire, quant à elle, privilégie ce que l'élève ressent, sa perception du monde, pour le transposer graduellement en texte, dans une sorte de mise en mots de l'imaginaire. Elle invite à accorder moins d'attention à la forme, à la construction logique, aux règles de la grammaire textuelle, en bref, aux contraintes. Il s'agit plutôt de se demander "comment faire en sorte que se développe chez l'élève la pensée singulière, comment faire en sorte qu'il pose un regard particulier sur le monde et qu'il puisse

5. MEQ, Programme de formation de l'école québécoise, ouvr. cité, p. 84

6. Yves Reuter, "Éléments de réflexion sur la place et les fonctions de la littérature dans la didactique du français à l'école primaire", Repères, Paris, $\mathbf{n}^{\circ} \mathbf{1 3}$ 1996, p. 7-25.

7. Karl Canvat, Enseigner la littérature par le genre. Pour une approche théorique et didactique de la notion de genre littéraire, Bruxelles, De BoeckDuculot, 1999. Pour Karl Canvat, "trois traits distinctifs caractériseraient la lecture littéraire. Le premier est l'attention à la polysémie du texte, réponse à sa "densité" constitutive. [...] Le deuxième trait de la lecture littéraire est sa fonction modélisante: la lecture littéraire propose au lecteur de vivre sur le mode imaginaire une expérience qu'il ne pourrait vivre dans la réalité. La troisième caractéristique de la lecture littéraire - peut-être la plus importante - est la dimension comparative : la lecture littéraire implique une compétence culturelle, qui permet de mesurer la part de conformité, d'innovation ou de subversion du texte [...]. (p. 111-112). 
l'écrire de manière tout aussi singulière ${ }^{*}$. Seulement, l'enseignement/apprentissage de l'écriture littéraire en classe de français doit également permettre aux productions littéraires de l'élève de tendre vers les caractéristiques de littérarité d'un texte".

Il est alors de la responsabilité des enseignants de proposer aux jeunes lecteurs non seulement des textes formateurs qui, tout en favorisant chez eux le goût de la lecture et des habitudes durables de lecture, développeront la connaissance qu'ils ont d'eux-mêmes et leur ouverture aux autres, mais aussi des textes qui leur permettront de se familiariser avec la littérature en les initiant aux ceuvres, de manière à les amener à témoigner de leur culture. Il importe de multiplier les contacts des élèves avec des cuvres de qualité appartenant ou non au répertoire classique de la littérature générale ou à celui de la littérature jeunesse. Cette posture tend à écarter les textes contraints à des règles strictes d'écriture imposées aux auteurs de littérature pour la jeunesse, tout comme ceux qui sont construits uniquement dans un but didactique ou encore tirés de manuels scolaires.

\section{Une littérature pour la jeunesse foisonnante}

Il existe de nos jours une pléthore de romans pour la jeunesse. Ils sont écrits par des adultes pour des enfants en âge d'apprentissage qui ne disposent pas toujours des mêmes outils cognitifs et référentiels que l'adulte pour percevoir et analyser le réel. L'auteur, "confronté à sa langue et à ses propres codes (sociaux et idéologiques, de manière consciente ou non) ${ }^{10}$ ", modèle son discours sur celui de son public cible, par souci de communication efficace. Il fait alors "l'effort d'utiliser un vocabulaire adapté au niveau supposé de conceptualisation, de raisonnement et de décodage de son lectorat ${ }^{11}$ ". Parfois, il arrive cepen-

8. Dominique Bucheton, "Vécu, affects et réécriture du récit ", Le français aujourd'bui, Paris, $n^{\prime 2} 108$, décembre 1994, p. 25.

9. Les caractéristiques d'un texte littéraire pourraient s'énumérer ainsi: a) l'appartenance à un genre, mais dont il se joue; b) l'intertextualité, dans un rapport dialogique avec dautres textes, d'autres discours; c) la création de sens originale lui conférant une certaine spécificité d) la création de sens élaborée, donc requêrant un contain travail sur le rexté, sur la langue.

10. Mireille Vagné-Lebas, "Littérature d'enfance et de jeunesse: formes et contenus", clans N. Zavialoff (sous la dir. de), La lecture. De la neurobiologie à la pédagogié, Paris, L'Harmattan, t. 1. 1990, p. 109.

11. Mireille Vagne-Lebas, "Littérature d'enfance et de jeunesse ", art. cité, p. 109. 
dant que le roman s'avère peu accessible au jeune lecteur, quand l'auteur opte pour "des codes de référence trop complexes ou inconnus ${ }^{12}$ ". Les enseignants constatent d'ailleurs que "certaines œuvres qu'ils "aiment", qui font partie de leur patrimoine culturel, ne "passent" plus auprès des élèves à cause de la difficulté de la langue ${ }^{13}$ " et de la barrière culturelle. Le texte crée alors chez le lecteur une certaine difficulté à appréhender ce que la lecture lui propose, celle-ci achoppant sans cesse sur le texte ${ }^{14}$. À l'inverse, dans certains textes pour enfants, le discours présente souvent des tendances infantilisantes ${ }^{15}$, le langage y est "bêtifiant " et le "style pauvre ${ }^{16 .}$. La lecture dépasse alors l'écriture, "parce qu'elle en connaît déjà la nature et le mode de fonctionnement ${ }^{17}$.

Heureusement, entre ces deux extrêmes, il existe de nombreux ouvrages de bonne qualité littéraire dont la langue et la construction narrative se révèlent d'une excellente tenue et qui n'ont rien d'infantilisant. En effet, si la clarté reste souhaitable, le texte et le lecteur peuvent aussi s'accommoder d'une certaine difficulté, la difficulté ne rimant pas "systématiquement avec hermétisme, ou illisibilité ${ }^{1 \%}$. La lecture trouve alors sa pleine mesure dans l'écriture qu'elle parcourt, et ce d'autant plus que les élèves de la fin du primaire ont clépassé le stade des premiers apprentissages. Ils sont déjà de "bons lecteurs" au sens du ministère de l'Éducation du Québec (MEQ) ${ }^{19}$, c'est-à-dire qu'ils sont capables de reconstruire le sens d'un texte avec le maximum d'aisance et de considérer la lecture comme un moyen de s'informer et de se divertir. Ils savent tenir compte du type de texte lu et de leur

12. Mireille Vagné-Lebas, "Littérature d'enfance et de jeuncsse" art. cité, p. 109.

13. Danièle Manesse, "Quels textes au collège?", La lettre de la DFIM (Didactique du français langue matemelle), Saint-Cloud (France), $\mathrm{n}^{\prime 2} 10,1992$, P. 9.

14. Ghislain Bourque, "Des mesures de lisibilité", dans Jean-Yves Boyer et Monique Lebrun (sous la dir. de), L'actualité de la recherche en lecture, Montréal, Association canadienne-française pour l'avancement des sciences (Actas). 1990 , p. $137-160$.

15. Dominique Demers, "Le héros sériel clans la littérature jeunesse québécoise" dans Hélène Charbonneau (sous la dir. de), Pour que vive la lecture. Littérature et bibliotbèques pour la jeunesse, Montréal, Association pour l'avancement des sciences et techniques de la documentation (Asted), 1994, p. 51-72.

16. Mireille Vagné-Lebas, "Littérature d'enfance et de jeunesse ", art. citế, p. 109.

17. Ghislain Bourque, "La matérialisation du lisible", Protée, Chicoutimi, vol. 14. $\mathrm{n}^{\mathrm{os}} 1-2,1986$, p. 94.

18. Mireille Vagné-Lebas, "Littérature d'enfance et de jeunesse", art. cité, p. 109.

19. MEQ, Programme d'études - Primaire - Français, Québec, Gouvernement du Québec, 1979. 
intention de lecture. Dès la fin du primaire, ils seront peut-être même devenus des "lecteurs avertis", habiles à saisir l'intention de l'auteur et à en critiquer aussi bien le fond que l'expression. Par ailleurs, pour qu'il y ait véritablement lecture, le rôle de l'enseignant consiste à privilégier auprès de ses élèves la rencontre avec des ouvres relevant davantage d'une esthétique littéraire que d'une pratique communicative, c'est-à-dire des œuvres qui comportent un certain degré de difficulté et s'ouvrent à la polysémie.

\section{Une littérature pour la jeunesse trop lisible}

Cependant, au début des années 1980 , avec la prise de conscience des problèmes de lecture chez les jeunes, s'est développé au Québec tout un courant de recherche soucieux d'interroger le degré de difficulté des textes qui leur sont destinés ou, si l'on préfère, leur lisibilité, en particulier dans les manuels scolaires. Dans le but de faire correspondre le langage de l'auteur à celui du lecteur, le Ministère de l'éducation a publié tour à tour plusieurs guides pour les auteurs de manuels scolaires ${ }^{20}$ et un guide de sélection du matériel didactique ${ }^{21}$. Les objectifs étaient, d'une part, de "sensibiliser l'auteur de manuel à certains facteurs susceptibles de jouer sur le degré de compréhension d'un texte" et, d'autre part, d'، indiquer, à l'aide de balises, dans quel sens certaines formes linguistiques peuvent être modifiées en faveur d'une compréhension optimale pour le lecteur visé ${ }^{22}$. Le ministère posait alors les jalons d'une lisibilité à la fois linguistique, rédactionnelle et typographique, accompagnés de critères rigides.

Un texte est dès lors lisible pour un lecteur donné, si celui-ci peut le lire sans trop de difficulté, s'il le comprend et l'intègre dans son ensemble. La lisibilité linguistique présume, entre autres, qu'un "texte est lisible pour un lecteur si les mots, les

20. MEQ, La lisibilité des textes destinés aux élèves du primaire. Guide pratique pour les auteurs de manuels scolaires, Québec, Gouvernement du Québec, 1981 ; La lisibilité des textes destinés aux élèves du secondaire. Guide pratique pour les auteurs de manuels scolaires, Québec, Gouvernement du Québec, 1983; La lisibilité rédactionnelle et typographique. Guide pratique pour les auteurs de manuels scolaires, Québec, Gouvernement du Québec, 1986.

21. MEQ, Guide destiné à la sélection du matériel didactique. La lisibilité dans les manuels scolaires, Québec, Gouvernement du Québec, 1983.

22. MFQ, La lisibilité des textes destinés aux élèves du secondaire, ouvr. cité, p. 3. 
phrases et le sens sont à sa portée, c'est-à-dire s'ils correspondent ou peuvent être reliés à sa réalité tant linguistique que culturelle ${ }^{23}$ ". La lisibilité s'inscrit alors "dans un vaste mouvement de simplification du langage dont le but général visé est l'efficacité de la communication, dans le sens d'une compréhension optimale ${ }^{24}{ }_{n}$, mouvement qui prône l'adaptation des textes à l'élève lecteur, c'est-à-dire au lecteur empirique.

L'histoire de la littérature pour la jeunesse montre que celle-ci aurait évolué selon ce même mouvement, passant d'un discours sur l'enfance à un discours à l'enfance ${ }^{25}$. En effet, d'une tradition didactique et édifiante qui caractérisait la littérature de jeunesse jusqu'au début des années 1970, alors que les ceuvres exprimaient "un regard d'adulte sur l'enfance ${ }^{26}$ "et que les héros étaient des modèles de vertu, on est passé à "une littérature ludique, constituée de héros miroirs" de l'enfant lecteur, où les ceuvres supposent "un regard d'enfant sur l'enfance". Toute la production pour la jeunesse est dès lors marquée par le roman socioréaliste, comme le constate Françoise Lepage ${ }^{27}$. Les représentations de l'enfance s'étant modifiées ces dernières décennies, on forge le héros à l'image "d'enfants réels, bien vivants et tout aussi imparfaits ${ }^{26}$ ", avec le souci de l'ancrer de plus en plus dans le quotidien. Une des tendances actuelles de la littérature pour la jeunesse vise donc le plaisir du lecteur et son identification immédiate au héros, qui a souvent son âge ou un peu plus et qui colle de près à ses rêves, à ses fantasmes, à ses aspirations. La littérature pour la jeunesse entend ainsi se faire complice du jeune lecteur.

À cette fin, les auteurs, souvent guidés par les maisons d'édition, se soumettent aux mêmes conditions de lisibilité que celles

23. MEQ, La lisibilité des textes destinés aux élètes du secondaire, ouvr. cité, p. 6.

24. MFQ, La lisibilité des textes destinés aux êlèves du secondaire, ouvr. cité, p. 79 .

25. Dominique Demers, "Discours à l'enfance et littérature jeunesse québécoise", Canadian Cbildren's Literature/Littérature canadienne pour la jeunesse (CCLLCJ), Guelph (Ontario), vol. 20, n³, automne 1994, p. 7-13.

26. Dominique Demers, "Le héros sériel dans la littérature jeunesse québécoise", art. cité, p. 71 .

27. Françoise Lepage, Histoire de la littérature pour la jeunesse, Orléans (Ontario), Éditions David, 2000 , p. 289.

28. Dominique Demers, "Le héros sêriel dans la littérature jeunesse québécoise ", art. cité, p. 55. 
qui, linguistiques, rédactionnelles ou typographiques, prévalent dans la rédaction des manuels scolaires ${ }^{29}$. Ces conditions impliquent, entre autres, des "indices d'intérêt humain", c'est-à-dire des mots personnels, un narrateur protagoniste à la première personne du singulier, des phrases rédigées en style direct ou encore des interpellations au lecteur. De plus, d'après Bruno Lemieux ${ }^{30}$, les maisons d'édition, en partie sans doute pour mieux courtiser le marché scolaire, appliquent les critères de qualité retenus par le ministère de l'Éducation ${ }^{31}$, qu'il s'agisse, notamment, de critères liés aux valeurs ou bien à la proximité du contenu. C'est pourquoi les cuvres véhiculent dorénavant "des valeurs d'amitié, de solidarité et de justice", invitent "à la compréhension et au respect de soi, des autres et du monde social et politique", "font appel au sens critique et [...] à la créativité des jeunes lecteurs". Tous les "éléments de racisme, de sexisme, de moralisme fermé, ceux qui font appel à une violence inutile, à une consommation aveugle, ceux qui versent dans l'infantilisme et la morale punitive. doivent être évacués des textes. Quant à la proximité de contenu, elle peut être d'ordre culturel, linguistique, thématique, psychologique, social, référentiel, etc., sans oublier naturellement un minimum d'ouverture destiné à permettre aux enfants "de tisser des liens entre le monde déjà connu [...] et les nouvelles expériences à faire".

En circonscrivant ainsi le profil du jeune lecteur, les auteurs et les maisons d'édition cherchent une plus grande lisibilité ou, du moins, une plus grande accessibilité. L'entreprise est louable, certes, mais les auteurs et les maisons d'édition, ce faisant, ont récupéré le roman pour la jeunesse en l'allégeant et en le simplifiant. Certains romans sont calibrés de façon mécanique ou adaptés d'ouuvres plus complexes. On a souvent l'impression, non plus qu'on crée des ceuvres, mais qu'on les construit suivant un impératif d'efficacité. Par le fait même, on nivelle la production des textes par le bas et on en appauvrit la qualité littéraire, prì-

29. Hélène Guy, "Ça se lit tout seul! Indices de tisibilité en littérature de jeunesse", Lurelu, Montréal, vol. 12, n” 3, hiver 1990, p. 2-6.

30. Bruno Lemieux, Le roman pour adolescents au Quêbec: édition normative et stratégies de mise en marché, mémoire de maîtrise, Université de Sherbrooke, 1994, p. 10-11.

31. MEQ, Littérature de jeunesse, Guide pédagogique, primaire, français, fascicule 2, Bibliographie sélective commentée, Québec, Gouvernement du Québec, 1981. 
vant ainsi les enseignants d'un bon outil au service de la didactique de la littérature. Et pourtant, tous ces romans sont on ne peut plus lisibles! Mais le lisible n'est-il pas précisément, comme le disait Roland Barthes, "ce qui peut être lu, mais non écrit "32"? Pour lui, les textes lisibles "sont des produits (et non des productions)": malheureusement, "ils forment la masse énorme de notre littérature" - et, pourrions-nous ajouter, de notre littérature pour la jeunesse.

Jean-Michel Adam insistait déjà sur cet aspect : "les récits "lisibles" de grande consommation usent de stéréotypes narratifs (situations toujours identiques) et de clichés dans les qualifications des lieux et des personnages $33_{n}$. Comme en écho, Dominique Demers dénonce de son côté certains romans en série qui, en littérature pour la jeunesse, utilisent "non seulement les mêmes personnages, mais des aventures et une trame narrative semblables", tels des "modèles photocopiables ${ }^{34}$ ". De même, les travaux de Bruno Lemieux montrent à quel point les romans québécois pour adolescents "tendent à ressembler le plus possible à un prototype [...] dont les caractéristiques standardisées facilitent la mise en marché ${ }^{35}$ ", de sorte qu'il faut bien en conclure qu'il s'agit là d'“édition normative. Françoise Lepage va même plus loin. Selon elle, "en l'absence de toute dimension symbolique, l'œuvre [pour la jeunesse] est ramenée à un simple bien de consommation immédiate, soumis aux modes, oublié sitôt consommé ${ }^{36}$. Outre les dangers inhérents à cette production industrielle du roman pour la jeunesse, on s'aperçoit, en somme, que le fait de poser la question du destinataire comme prioritaire a entraîné une déperdition du langage et un appauvrissement de la qualité littéraire des ouvres.

L'esthétique de la littérature pour la jeunesse serait donc devenue une esthétique de la communication en vertu de laquelle l'auteur est tenu d'ajuster son texte au lecteur en se

32. Roland Barthes, $S / Z$, Paris, Seuil, coll. "Tel Quel ", 1970, p. 10-11.

33. Jean-Michel Adam, Le texte narratif. Traité d'analyse textuelle des récits, Paris, Nathan, 1985, p. 121.

34. Dominique Demers, "Le héros sériel dans la littérature jeunesse québécoise", art. cité, p. 52.

35. Bruno Lemieux, Le roman pour adolescents au Québec, ouvr. cité, p. 185186.

36. Françoise Lepage, Histoire de la littérature pour la jeunesse, ouvr. cité, p. 337. 
pliant aux exigences du marché. Malgré des efforts louables pour rendre la littérature accessible au plus grand nombre, il faut désormais se rendre au constat suivant: plutôt que d'amener les jeunes à la littérature, on a amené la littérature aux jeunes en opérant une sorte de nivellement par le bas du fait littéraire.

Dès lors, on perd évidemment de vue les ceuvres de qualité que souhaite obtenir pourtant le MEQ en classe de français lors des pratiques de lecture littéraire. L'enseignant est plutôt placé devant le répertoire immense des ouvres destinées à la jeunesse, répertoire variable tant en difficulté qu'en qualité. Il se retrouve, en particulier, submergé par une kyrielle de romans, faciles à lire certes, mais qui ne correspondent pas toujours à la qualité qu'exige le souci de travailler la langue et le texte selon l'optique d'une lecture et d'une écriture qui se veulent littéraires.

paradoxalement, on exige trop peu des élèves des $2^{e}$ et $3^{c}$ cycles du primaire - et encore moins du $1^{\text {er }}$ cycle du secondaire - en matière de lecture littéraire. Quant à l'écriture littéraire - qui dépasse de beaucoup la simple écriture de textes narratifs ou de textes de fiction -, elle est la grande absente des pratiques scolaires. Certes, les élèves n'ont sans doute jamais autant lu de romans, mais des romans par trop accessibles, caractérisés par leur simplicité et leur naîveté thématique. Plus qu'un livre de loisir, le roman pour la jeunesse pourrait être utilisé par l'enseignant à des fins pédagogiques et de manière à soutenir, dès le primaire, un travail véritablement littéraire. Il lui faut donc des ceuvres pourvues d'une littérarité certaine pour pouvoir en reconstruire le parcours interprétatif, des ouvres où la lecture trouve sa pleine mesure dans l'écriture, des ceuvres, en somme, qu'on n'a pas adaptées au lecteur afin de mieux se soumettre à un impératif de communication, voire de consommation.

\section{Le Lecteur modèle et le lecteur empirique}

Après avoir tracé ce portrait assez sombre d'une littérature pour la jeunesse, littérature lisible certes, mais qui répond mal aux besoins des enseignants en matière d'apprentissage littéraire et qui pose la question cruciale du choix de textes littéraires à exploiter en classe, quelles avenues proposer à la réflexion? Le volet culturel du nouveau curriculum réserve une place de choix à l'exploration et à l'appréciation d'œuvres littéraires nombreuses et variées, et souligne l'importance d'un corpus riche, issu "de la 
littérature pour la jeunesse ou de la littérature générale accessible aux jeunes ${ }^{37} \%$. Il s'agit donc ici d'œuvres non calibrées et non normées, de textes où le Lecteur modèle ne se résoudrait pas au lecteur empirique, bien au contraire: le rôle de l'enseignant serait même d'amener ce lecteur empirique à se réaliser comme Lecteur modèle.

Le Lecteur modèle, comme nous l'indiquions plus haut, renvoie à cette stratégie textuelle qui correspond au rôle attendu du lecteur, au rôle que lui prescrit le texte, rôle vers lequel doit tendre le lecteur empirique. Le Lecteur modèle rëfère donc à l'ensemble des opérations exigées par le texte pour être activé. En premier lieu, le texte se manifeste dans sa surface linéaire, et c'est en partant de cette manifestation que le lecteur entame son travail d'analyse et d'interprétation. Dans un second temps, afin d'éventuellement construire l'histoire grâce à ses propres connaissances encyclopédiques d'une part, à un travail inférentiel complexe d'autre part, le lecteur est appelé à supposer et à actualiser le contenu des structures et des divers niveaux: celles-là sont narratives ou discursives, ceux-ci ne sont hiérarchisés ni temporellement ni logiquement, mais étroitement corrélés.

Quand un auteur produit un texte, il tient compte de l'interprétation dont son texte sera l'objet: "Générer un texte signifie mettre en œuvre une stratégie dont font partie les prévisions des mouvements de l'autre ${ }^{38_{n}}$. Il se doit donc d'organiser sa stratégie textuelle en se basant sur un ensemble de compétences qui fourniront un contenu à son choix d'expressions. Cependant, il vise à ce que l'ensemble de ces compétences auxquelles il se réfère corresponde à celles de son lecteur, d'où la nécessité pour l'auteur de prévoir "un Lecteur modèle capable de coopérer à l'actualisation textuelle de la façon dont lui, l'auteur, le pensait et capable aussi d'agir interprétativement comme lui a agi générativement ${ }^{39}$ ". En effet, prévoir son Lecteur modèle ne signifie pas uniquement savoir qu'il existe, mais signifie aussi travailler le texte de façon à construire ce lecteur: «L'auteur présuppose la compétence de son Lecteur Modèle et en même temps il l'institue ${ }^{40}$. Un texte

37. MEQ, Programme de formation de l'école québécoise, ouvr. cité, p. 84.

38. Umberto Eco, Lector in fabula, ouvr. cité, p. 65.

39. Umberto Eco, Lector in fabula, ouvr. cité, p. 68.

40. Umberto Eco, Lector in fabula, ouvr. cité, p. 68. 
s'appuie non seulement sur une compétence, mais il contribue également à l'élaborer.

Un texte, rappelons-le, est un tissu de "non-dit ${ }^{41}$ ". Le rôle du lecteur est d'actualiser ce non-dit à travers un ensemble complexe de mouvements coopératifs. Le texte se réserve des espaces blancs à suturer ${ }^{42}$, qui laissent au lecteur toute initiative quant à l'interprétation, même si, en général, le texte n'est interprétable qu'avec une marge suffisante d'univocité. Un texte postule son lecteur, mais il n'est absolument pas nécessaire que ce destinataire existe concrètement ou empiriquement. Il peut se mouvoir dans la seule immanence, et s'y situer uniquement au plan du Lecteur modèle.

Toutefois, ce travail de suture, ce travail sémiotique d'interprétation que le texte exige de son lecteur soulève la question de la compétence, notamment, celle du lecteur empirique. Ce système complexe qu'Umberto Eco définit comme étant la "compétence encyclopédique "englobe toutes les connaissances nécessaires à la construction de la signification du texte et sert d'assise à l'activité inférentielle du lecteur. On peut donc présumer que l'auteur a en tête un lecteur empirique possédant une connaissance encyclopédique et une certaine compétence littéraire. Ce lecteur empirique, comme sujet concret du processus de lecture, doit devenir conforme ou tendre vers le Lecteur modèle.

En effet, si le Lecteur modèle représente "un ensemble de conditions de succès ${ }^{43}$ ", c'est au lecteur empirique de réaliser ces conditions à travers l'activité pragmatique qu'est la lecture. Le lecteur empirique, lors de ces exécutions concrètes qui lui permettent de se confronter au Lecteur modèle, met en jeu ses connaissances antérieures qui constituent, en quelque sorte, son encyclopédie, pour reprendre l'expression d'Umberto Eco, et plus particulièrement sa compétence littéraire. Le Lecteur modèle sert donc d'ancrage, et limite ainsi le travail interprétatif du lecteur empirique.

L'auteur va cerner son Lecteur modèle à partir de textes ouverts, polysémiques, invitant une lecture plurielle, ou encore de textes fer-

41. Oswald Ducrot, Dire et ne pas dire. Principes de sémantique linguistique, Paris, Hermann, 1972.

42. Lucien Dällenbach, "La lecture comme suture", dans Lucien Dällenbach et Jean Ricardou (sous la dir. de), Problemes actuels de la lecture, Paris, Clancier-Guenaud, 1982, p. 35-47.

43. Umberto Eco, Lector in fábula, ouvr. cité, p. 77. 
més, n'offrant qu'un seul parcours de lecture, selon qu'il cible, aux deux extrêmes, des érudits ou des lecteurs dits naifs. Il choisit alors un certain type d'encyclopédie, un certain dictionnaire lexical, phrastique ou discursif. D'un côté, le lecteur naîf n'évite aucun des pièges tendus par le texte; de l'autre, le lecteur critique est "en mesure de reconstruire le parcours de sa propre lecture de la façon suggérée et requise par le texte ${ }^{4 *_{*}}$. Ces deux lecteurs diffèrent non seulement dans leurs activités, mais aussi dans leur compétence de lecteur. Puisque nous nous intéressons ici à la littérature pour la jeunesse, notre Lecteur modèle est plutôt de type naif, "cette stratégie s'inscrivant dans un processus de lecture linéaire ${ }^{45}$ ", lequel s'oppose à la lecture plurielle associée à une lecture plus littéraire.

\section{Le rôle de l'enseignant}

Lorsqu'un enseignant veut utiliser le roman pour la jeunesse à des fins pédagogiques, il ne s'intéresse pas seulement à sa complexité linguistique linéaire et de surface: il doit encore recentrer son intervention sur les caractéristiques du texte et de son interprétation, c'est-à-dire qu'il doit découvrir comment un texte dit ce qu'il a à dire et comment il prévoit son propre lecteur. Il importe donc de savoir comment le rôle du lecteur y est inscrit et comment sa participation est pressentie aux différents niveaux de la structure textuelle. On sait, en effet, que chaque texte construit son Lecteur modèle.

Un tel fait met en évidence la nécessité d'une didactique de la littérature au primaire et l'importance, pour la formation des futurs enseignants et le perfectionnement des enseignants en exercice, de l'élaboration d'un programme en littérature et en didactique de la lecture et de l'écriture littéraires. La littérature et sa didactique devraient tenir compte d'une complexité globale qui touche à la fois au texte et à ses différents niveaux de structures, et à son interprétation déjà inscrite dans la stratégie textuelle qui institue le Lecteur modèle, stratégie qui contraint la production de sens du lecteur empirique. Cet écart entre le lecteur empirique et le Lecteur modèle peut se résorber grâce à l'apprentissage, et le rôle de l'enseignant consiste précisément à aider le lecteur empirique à se réaliser comme Lecteur modèle. Il lui

44. Patrizia Violi, "Du côté du lecteur ", Versus, Milan, $n^{* *} 31 / 32,1982$, p. 8.

45. Patrizia Violi, "Du côté du lecteur", art. cité, p. 12. 
faudra donc établir les compétences et les connaissances que cette coopération exige du lecteur qui est tenu de satisfaire, à travers le processus de lecture, les conditions de succès que représente le Lecteur modèle. Il lui faudra faire se conjoindre le lecteur empirique et le Lecteur modèle, en proposant des opérations cognitives et langagières, et des stratégies pertinentes à l'apprentissage du jeune lecteur, pour qu'il ressente le littéraire et s'en inspire dans ses propres productions. Certes, nous sommes loin d'un projet didactique opérationnel, et nous nous sommes surtout attachée ici à appréhender les enjeux liés aux biens que comporte la littérature pour la jeunesse, tout en soulignant leur étroite relation avec la lecture littéraire.

\section{Conclusion}

Le volet culturel du nouveau programme réserve dorénavant une place privilégiée à l'exploration et l'appréciation des oeuvres littéraires. Toutefois, cela pose la question cruciale du choix des ceuvres à exploiter en classe et met en évidence la nécessité de former en didactique de la littérature les enseignants et les futurs enseignants du primaire.

Après la relance fulgurante de la littérature québécoise pour la jeunesse, qui a marqué la fin du $x^{2}$ siècle, relance remarquable du point de vue de la quantité, nous recherchons dorénavant une plus grande qualité. La littérature pour la jeunesse, longtemps conçue comme appartenant à la fois au système éducatif et au système littéraire, reprendrait alors ses droits en littérature. Or, comme l'affirme Françoise Lepage, "elle ne parviendra réellement à cette reconnaissance qu'en enrichissant le contenu des ouvres, tant sur le plan humain (approfondissement de l'analyse psychologique, par exemple), que sous l'angle de l'écriture ou de la polysémie, en donnant des ouvres à résonances multiples [... ${ }^{4}{ }^{4}$.

Rendre le roman plus accessible, donc plus lisible, ne devrait pas revenir à l'auteur, ni à l'éditeur, mais à l'enseignant qui doit mettre en lumière ses éléments constitutifs et les travailler avec le jeune lecteur de manière à réduire l'écart entre lecteur empirique et Lecteur modèle.

46. Françoise Lepage, Histoire de la littérature pour la jeunesse, ouvr. cité, p. 337 . 
Travailler sur la complexité des textes, à partir d'œuvres pertinentes par leur littérarité, serait donc essentiel à l'apprentissage de la langue et à la structuration de la pensée, mais également à l'acquisition d'une compétence littéraire qui se construit dès le plus jeune âge. Cette posture pédagogique aurait des incidences sur la sélection des romans à faire lire aux élèves et, en contrepartie, sur la qualité des romans pour la jeunesse. En effet, les maisons d'édition de livres pour la jeunesse et, par le fait même, les auteurs pourraient s'affranchir de cette tendance à produire des romans édulcorés, des ceuvres résolument lisibles, non pas conçues dans une optique littéraire, mais communicative, où l'auteur doit avant tout tenir compte de la réception de son ouvre en l'adaptant au jeune lecteur. Les espaces vides, le non-dit du texte se trouvent ainsi comblés par l'auteur qui tente d'atteindre le plus haut degré de prévisibilité, comme s'il interprétait déjà le texte pour le lecteur. En évacuant toute ambiguité du texte, toute lecture plurielle, c'est-à-dire différents parcours d'interprétation, l'auteur prive son ceuvre de sa dimension littéraire et artistique. De même, en cédant aux pressions du marché, les maisons d'édition nous offrent trop souvent une littérature stéréotypée, normalisée et banale. Ce n'est pourtant qu'une fois libérée de ces contingences que la littérature pour la jeunesse pourrait enfin être considérée comme une forme autonome d'expression. 